\title{
THE ISO PERSPECTIVE ON NORMAL GALAXIES
}

\author{
G. HELOU \\ Infrared Processing and Analysis Center, Caltech \\ Pasadena CA 91125, USA
}

\section{Introduction}

Based on results from the first half of its mission, ISO (Kessler et al. 1996) is contributing to our understanding of normal galaxies by offering effective gauges of the physical conditions within the interstellar medium (ISM) of galaxies. ISO data permit a more precise and detailed characterization of the radiation, dust and gas deep within many more galaxies than had been previously possible. This in turn yields new insight into the regulation mechanisms of star formation and galaxy evolution. Apart from these ISM diagnostics, ISO also contributes a vastly improved empirical de-m scription of galaxies at all infrared wavelengths, which will be critical in guiding the search for the origins and early life of galaxies.

\section{Line Spectroscopy}

Using the ISO-SWS (de Graauw et al. 1996), Valentijn et al. (1996) reported the first detection of $\mathrm{H}_{2}$ in galaxies, and derived temperature, mass and density of the emitting molecular material based on three transitions. Most other molecular line work to date is associated with starburst galaxies and covered by Alan Moorwood's review in this volume.

$[\mathrm{C} \mathrm{II}]$ and $[\mathrm{O} \mathrm{I}]$ are well known as the main gas cooling lines in photo-dissociated regions (PDR), the warm transition layer between molecular and ionized material in star formation regions. They were well studied with the KAO in bright Milky Way regions and in starburst galaxies. The ISOLWS (Clegg et al. 1996) is building on KAO results in this area, and reaching well beyond the KAO in terms of sensitivity and redshift to probe the ISM in a wide variety of normal galaxies.

Two thirds of normal galaxies have $\mathrm{L}_{[\mathrm{C} \mathrm{I}]} / \mathrm{L}_{\mathrm{FIR}}$ in the range $2-7 \times 10^{-3}$ and $\mathrm{L}_{[\mathrm{O} \mathrm{I}]} \simeq \mathrm{L}_{[\mathrm{C} \mathrm{I}]}$ to within a factor of three roughly (e.g. Lord et al. 1996), consistent with KAO data, the physics of photoelectric heating of gas, and detailed PDR theories (Tielens 1995). The remaining, more active galaxies display a trend of decreasing $\mathrm{L}_{[\mathrm{C}}{ }_{\mathrm{I}} / \mathrm{L}_{\mathrm{FIR}}$ with increasing $f_{\nu}(60 \mu \mathrm{m}) / f_{\nu}(100 \mu \mathrm{m})$ dust temperature, and with increasing optical depth as measured by the ratio of far-infrared to blue band luminosity $\mathrm{L}_{\mathrm{FIR}} / \mathrm{L}_{\mathrm{B}}$ (Malhotra et al. 1997). This decrease by $\times 10-40$ could be caused by either or both of: reduced efficiency of photoelectric heating at higher UV intensities, or self-absorption of $[\mathrm{C} \mathrm{II}]$ in the cooler outer layers of the system. More active star formation is associated with greater UV intensities and greater optical depths (Wang \& Helou 1992), so an increasingly significant AGN is not a necessary hypothesis.

Smith \& Madden (1997) have compared [CII] and CO line fluxes in five low-mass spiral galaxies, and found that in two cases much of $\mathrm{L}_{[\mathrm{C} \mathrm{r}]}$ could originate in "warm neutral medium" $\mathrm{HI}$ at $n \sim 90 \mathrm{~cm}^{-3}$ and $\mathrm{T} \sim 80 \mathrm{~K}$. These two galaxies have abnormally high $[\mathrm{CII}] / \mathrm{CO}$ ratios, but normal $\mathrm{L}_{\left[\mathrm{C}_{11}\right]} / \mathrm{L}_{\mathrm{FIR}}$ ratios, pointing to a much depleted molecular medium and a relatively diffuse PDR.

\section{Mid-Infrared Continuum}

ISO-CAM (Césarsky et al. 1996) has obtained striking images of galaxies between 4 and $18 \mu \mathrm{m}$, showing dust emission in nuclear regions, outlining the spiral arms, and tracing the disk out to the Holmberg radius and beyond (Malhotra et al. 1996, Sauvage et al. 1996, Boselli \& Lequeux 1997, Smith 1998). There are clear color variations within spiral galaxies, some of which have not 
yet found satisfactory explanations (Helou et al. 1996; Vigroux 1997). Rouan et al. (1996), Block et al. and Smith (1998) have combined mid-infrared and $\mathrm{Br} \gamma$ images with other broad-band and line images to estimate star formation rates, ISM parameters, obscuration and dust properties. These studies generally point to aromatic feature carriers, generally described as large molecules with benzene rings, as a ubiquitous component of interstellar dust, to likely destruction of these carriers at high UV intensities, and to dust heating being derived from both old stars and OB stars in non-starburst spiral galaxies.

Mid-infrared colors derived from ISO-CAM are effective discriminants between stellar photospheres and dust emission. Lenticular galaxies often have contributions from both os those sources (Madden et al. 1997). The ratios of mid-infrared to far-infrared colors are still being analyzed for indications of aromatic carrier destruction at high UV intensities (Boselli et al. 1997). The 7-to-15 $\mu \mathrm{m}$ color ratio drops sharply when heating intensifies to the point where classical dust temperature exceeds $100 \mathrm{~K}$, which happens at an intensity a thousand times greater than the diffuse interstellar radiation field in the local Milky Way (Helou et al. 1997). At these intensities the classical dust blackbody emission starts dominating the $15 \mu \mathrm{m}$ band with the sharp rise of the Wien tail of the blackbody curve, whereas aromatic carrier emission in the $7 \mu \mathrm{m}$ band is either rising linearly with heating intensity, or decreasing because of carrier destruction.

\section{Mid-Infrared Spectroscopy}

Star forming galaxies all have similar mid-infrared spectra as revealed by ISO-PHOT-S (Lu et al. 1996), CAM-CVF (Metcalfe et al. 1996), and SWS. Exceptions arise in the presence of AGN or Seyfert nuclei, as in Cen A (Vigroux 1997). Aromatic features and plateaus carry $50-75 \%$ of the luminosity between 3 and $12 \mu \mathrm{m}$, and $5-20 \%$ of $\mathrm{L}_{\mathrm{FIR}}$. Feature-to-feature ratios are largely constant, except that the $11.3 \mu \mathrm{m}$ feature tends to be stronger in more quiescent galaxies with cooler far-infrared colors.

The underlying 5 to $12 \mu \mathrm{m}$ continuum is flat and independent of far-infrared colors. Silicate absorption at $10 \mu \mathrm{m}$ is not prominent except in very active galaxies. In light of the new full-range PḢ-S spectra, it is necessary to revise the optical depths derived from ground-based silicate absorption estimates (Roce et al. 1991).

\section{Far-Infrared Mapping}

ISO-PHOT (Lemke et al. 1996) is building on IRAS results, obtaining maps with finer pixels, and extending the wavelength coverage from $100 \mu \mathrm{m}$ to $200 \mu \mathrm{m}$ (Tuffs et al. 1996). Hippelein et al. (1996) have analyzed PHOT maps of M101 at 60,100 and $175 \mu \mathrm{m}$ for spatial variations in the spectral energy distribution. They find little variation açoss the disk, except where HII region complexes appear bright and warm. $L_{F I R}$ is mostly derived from dust heated by the diffuse interstellar radiation field, emitting with a color temperature $\mathrm{T} \sim 24-28 \mathrm{~K}$, while $\sim 15 \%$ of $\mathrm{L}_{\mathrm{FIR}}$ comes from dust at $\mathrm{T} \sim 28-33 \mathrm{~K}$, directly heated by OB stars. This is direct evidence that in non-starburst galaxies $\mathrm{L}_{\mathrm{FIR}}$ is not simply proportional to the "current star formation rate", a conclusion derived from IRAS data but occasionally controversial (Helou 1986, Devereux \& Scowen 1994).

\section{Conclusion}

These varied ISO data will permit a better understanding of star formation in galaxies, the main evolutionary mechanism in the local Universe, and a critical key to much of the history of the Universe. A better description of the relation between quiescent and more active systems will lead to greater insight into the most extreme star-forming systems, starbursting and ultraluminous galaxies. Such objects are the most promising probes of the early Universe, and may well be the precursors of quasars.

ISO is an ESA project with instruments funded by ESA member states, with participation by ISAS and NASA. This work was supported by ISO data analysis funding from the National Aeronautics and Space Administration, and was carried out at the Infrared Processing and Analysis Center and the Jet Propulsion Laboratory of the California Institute of Technology. 


\section{References}

Roche, P.F. et al 1991, MNRAS, 248, 606.

Block, D.L. et al. 1997, ApJL, 486, L95.

Boselli, A., Lequeux, J., Contursi, A. et al. 1997, A\&A, 324, L13.

Boselli, A. \& Lequeux, J. 1997, in Rencontres de Moriond 1997.

Césarsky, C. et al. 1996, A\&A, 315, L32.

Clegğ, P.E. et al. 1996, A\&A, 315, L38.

de Graauw, T. et al. 1996, A\&A, 315, L49.

Devereux, N.A. \& Scowen, P.A. 1994, AJ, 108, 1244.

Helou, G. 1986, ApJ, 311, L33.

Helou, G. 1997, in preparation.

Helou, G., Malhotra, S., Beichman, C.A. et al. 1996, A\&A, 315L, 157.

Hippelein, H., Lemke, D., Haas, M. et al. 1996, A\&A, 315, L82,

Kessler, M.F. et al. 1996, A\&A, 315, L27.

Lemke, D. et al. 1996, A\&A, 315, L64.

Lord, S.D., Malhotra, S., Lim, T. et al. 1996, A\&A, 315, L117.

Lu, N., Helou, G., Beichman, C.A. et al. 1996 BAAS, $28,1356$.

Madden, S., Vigroux, \& Sauvage 1997, in Rencontres de Moriond 1997.

Malhotra, S., Helou, G., Van Buren, D. et al. 1996, A\&A, 315, L161.

Malhotra, S., Helou, G. Stacey, G. et al. 1997, ApJ, 491, L27.

Metcalfe, L., Steel, S.J., Barr, P. et al., 1996, A\&A, 315, L105.

Rouan, D., Tiphène, D., Lacombe, F. et al. 1996, A\&A, 315, L14.

Sauvage, M., Blommaert, J., Boulanger, F. et al. 1996, A\&A, 315, L89.

Smith, B.J. \& Madden, S.C. 1997, AJ, 114, 138.

Smith, B.J. 1998, submitted.

Tielens, A.G.G.M. 1995, ASP Conference Series, 73, 3.

Tuffs, R., Lemke, D., Xu, C. et al. 1996, A\&A, 315, L149

Valentijn, E.A., et al. 1996, A\&A, 315, L145.

Vigroux, L. 1997, private communication.

Wang, Z. \& Helou, G. 1992, ApJL, 398, L33. 